\title{
Effectiveness of Solution with 5\% Detergent for Cleaning Transnasal Esophagogastroduodenoscopy Lens
}

\author{
Yoshinori Komazawa', Mika Yuki', Nobuhiko Fukuba ${ }^{2}$, Yoshiya Kobayashi ${ }^{2}$, Hitomi Ishitobi ${ }^{2}$, Sayaka Nakashima', Makoto \\ Nagaoka ${ }^{2}$, Yoshiko Takahashi' and Toshihiro Shizuku ${ }^{2}$
}

${ }^{1}$ Department of Gastroenterology, Izumo Tokushukai Hospital, ${ }^{2}$ Division of Internal Medicine, Izumo City General Medical Center, Izumo, Japan

Background/Aims: Unsedated transnasal esophagogastroduodenoscopy (EGD) is affected by a poor scope lens-cleaning function. We have previously reported good, albeit limited, effects of an oolong tea washing solution; here, we evaluated the effectiveness of a $5 \%$ lens cleaning solution for cleaning an EGD lens.

Methods: Five percent lens cleaning solution (C), 5\% dimethicone solution (D), and distilled water (W) were prepared. Study I: Lenses were soiled with pork grease, washed with each washing solution, and their image quality was judged. Study II: Patients ( $n=996)$ scheduled for transnasal EGD were randomly assigned to the C- or W-group. Lens cleanliness level, washing solution volume used, and endoscopist stress due to lens contamination were determined.

Results: Study I: The image quality of the lenses washed with (C) was significantly superior. (D) was clinically unsuitable because of spray nozzle clogging. Study II: Lens cleaning in the C-group was significantly superior $(p<0.0001)$ and the solution volume required was significantly reduced $(p<0.0001)$, while endoscopist stress was also lower $(p<0.0001)$.

Conclusions: For transnasal small-caliber EGD, the present 5\% lens cleaning solution provided good visibility. It features a high detergency level and is simple to formulate for therapeutic endoscopy applications, such as endoscopic submucosal dissection. Clin Endosc 2021;54:236-241

Key Words: Lens cleaner; Small-caliber endoscope; Transnasal esophagogastroduodenoscopy; Visual quality

\section{INTRODUCTION}

Unsedated transnasal esophagogastroduodenoscopy (EGD) has been proven to be useful for examinations of the upper gastrointestinal tract with good feasibility and tolerability. The use of a small-caliber endoscope decreases choking sensations and gagging episodes; hence, it is less stressful to the digestive system of the patient. ${ }^{1-6}$ However, a limitation of transnasal

Received: February 27, 2020 Revised: June 8, 2020

Accepted: August 20, 2020

Correspondence: Yoshinori Komazawa

Department of Gastroenterology, Izumo Tokushukai Hospital, 3964-1 Naoe, Hikawa-cho, Izumo, Shimane 699-0631 Japan

Tel: +81-853-70-7000, Fax: +81-853-70-7077, E-mail: komazawa@384.jp

ORCID: https://orcid.org/0000-0002-4002-3245

cc This is an Open Access article distributed under the terms of the Creative Commons Attribution Non-Commercial License (http://creativecommons.org/ licenses/by-nc/3.0) which permits unrestricted non-commercial use, distribution, and reproduction in any medium, provided the original work is properly cited.
EGD is its poor scope lens cleaning function due to the weak spraying of the washing solution and aspiration of fluid compared with a conventional endoscope. .10 $^{\text {-10 }}$

An unclear endoscopic view of the target field can adversely affect accurate endoscopic diagnosis. Previously, we reported the usefulness of oolong tea, which contains saponins with surfactant action, as a lens cleaning solution for transnasal $\mathrm{EGD},{ }^{7}$ although the effects were limited and sometimes inadequate for routine clinical applications. ${ }^{11,12}$

A new type of lens cleaner (Cleash ${ }^{\circledR}$; Fujifilm Co., Tokyo, Japan), which contains nonionic harmless surfactants became available for purchase in Japan in 2015. Although it was recently reported that a very low concentration $(0.5 \%)$ of Cleash solution improved the adherence of water droplets to the endoscope lens, ${ }^{12}$ its lens cleaning ability has yet to be examined. In this study, we evaluated the efficacy of a lens cleaning solution consisting of $5 \%$ Cleash solution for transnasal EGD with a small-caliber endoscope in both basic and clinical examinations. 


\section{PATIENTS AND METHODS}

We initially determined the optimum concentration of the new lens cleaner (Cleash; Fujifilm) for use as a washing solution. To obtain a high cleaning effect, we determined that a high concentration of Cleash solution would be needed. However, with higher concentrations, inconvenient bubbles were generated through the endoscopic spray from the water-jet nozzle. To counteract this effect, we added dimethicone as a washing solution to reduce the bubbles. Additionally, we tested various concentrations of Cleash and dimethicone, and finally decided to adopt a $5 \%$ concentration, which was obtained by adding $10 \mathrm{~mL}$ of Cleash and $10 \mathrm{~mL}$ of dimethicone to $180 \mathrm{~mL}$ of distilled water.

An in vitro study (Study I) and a randomized prospective clinical study (Study II) were subsequently performed to evaluate the efficacy of the $5 \%$ Cleash solution for cleaning the lens of small-caliber endoscopes used for transnasal EGD examinations.

\section{Study I}

Using artificially soiled lenses of small-caliber endoscopes, we evaluated the cleaning effect by determining the image quality of photographs obtained after endoscopic lens cleaning procedures. Three washing solutions, namely, a 5\% Cleash solution, obtained by adding $10 \mathrm{ml}$ of Cleash and $10 \mathrm{~mL}$ of dimethicone to $180 \mathrm{~mL}$ of distilled water (C), a 5\% dimethicone solution, obtained by adding $10 \mathrm{~mL}$ of dimethicone to 190 $\mathrm{mL}$ of distilled water (D), and distilled water only (W), were used for cleaning the lens of a small-caliber endoscope (GIFXP260N; Olympus Medical Systems Co., Tokyo, Japan).

First, the tip of the endoscope was immersed in pork grease heated to $37^{\circ} \mathrm{C}$. Then, photographs of 2 different test charts were obtained after a lens-cleaning procedure that consisted of ejection of each washing solution and subsequent air from the endoscopic jet nozzle for 5 seconds each. Photographs were obtained after every soiling and cleaning procedure, with a total of 12 procedures performed with each cleaning solution. We used pork grease as the soiling material because of its turbid and sticky characteristics similar to those of lipids and proteins. Furthermore, we considered that pork grease resembles lens contamination caused by high frequency devices used during therapeutic endoscopy procedures such as endoscopic submucosal dissection (ESD).

The quality and clarity of the photographs was evaluated. Photographs were taken at a distance of $5 \mathrm{~cm}$ from each test chart. Image quality was evaluated by 7 expert endoscopists with no prior knowledge of the washing solution used, with the results categorized into 5 levels as follows: over $50 \%$ of the field is unclear (level 1), clarity midway between level 1 and 3 (level 2), approximately $80 \%$ of the field is clear (level 3), clarity midway between levels 3 and 5 (level 4), the entire field is clear (level 5). Representative photographs from each group are shown in Fig. 1. Based on our results, we judged that (D) was

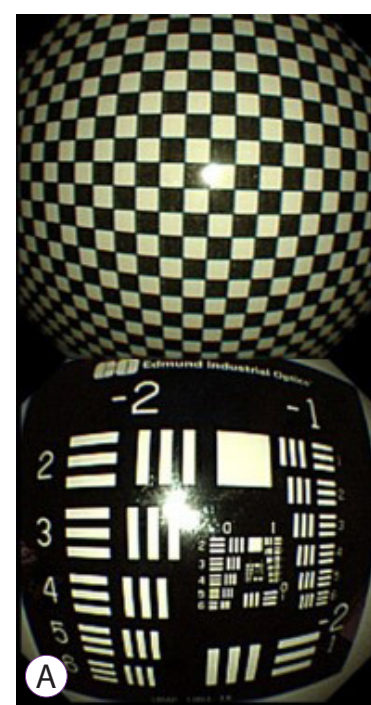

Level 5

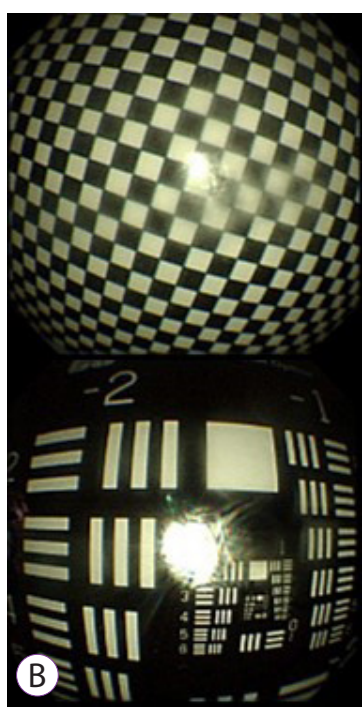

Level 3

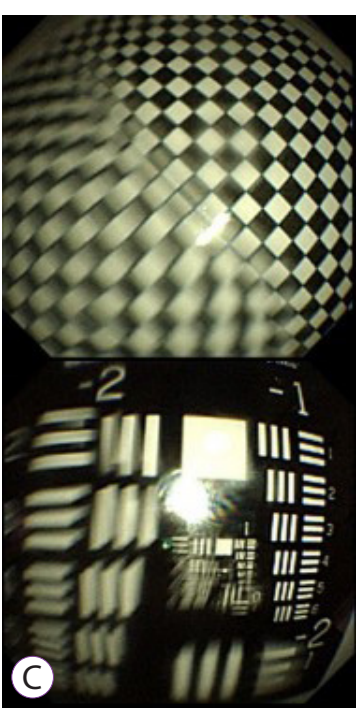

Level 1

Fig. 1. Photographic images of two different test charts observed through the lens of a (GIF-XP 260N; Olympus, Tokyo, Japan) endoscope soiled by pork grease and then subjected to cleaning. (A) Level 5 cleanliness. (B) Level 3 cleanliness. (C) Level 1 cleanliness. 
not suitable for clinical use because obstruction of the spray nozzle frequently occurred. Finally, we decided to use (C) and (W) (control) for the clinical study of the washing solutions.

\section{Study II}

For clinical evaluation of the efficacy of the (C) for lens cleaning, we performed a randomized controlled prospective study. Patients $(n=996)$ scheduled to undergo unsedated transnasal EGD for abdominal symptoms or an annual medical check between July and September 2017 at Izumo City General Medical Center, Shimane, Japan were enrolled. Those who underwent upper gastrointestinal tract surgery were excluded from the study. The diameter of the small-caliber endoscope used was 5.9 mm (EG-580NW2 or EG-L580NW; Fujifilm) or $5.5 \mathrm{~mm}$ (GIF-XP 260N; Olympus, Tokyo, Japan), with the type randomly selected for each procedure. Patients were randomly assigned to the $\mathrm{C}$-group $(10 \mathrm{~mL}$ of Cleash and 10 $\mathrm{mL}$ of dimethicone in $180 \mathrm{~mL}$ of distilled water) or W-group (distilled water only). The washing solutions were placed in a covered washing solution tank by the medical support staff, while the endoscopist was blinded to the solution used. The color of each of the washing solutions were also such that the endoscopist could not distinguish between them during the endoscopy procedure.

Three aspects of each procedure were evaluated: the volume of washing solution used during the examination, stress level of the attending endoscopist due to lens contamination, and level of cleanliness of the endoscopic lens. The volume of washing solution used was determined by measuring the volume remaining in the tank at the end of each examination. Immediately after EGD, the endoscopist judged the level of stress due to lens contamination and the level of cleanliness during the procedure using five grades: level 1 (very stressful, impossible to provide accurate diagnosis), level 2 (between levels 1 and 3), level 3 (normal, possible to diagnose but with some difficulties), level 4 (between levels 3 and 5), and level 5 (no stress, fully satisfied with quality of the image).

All the procedures were performed by expert gastroenterologists experienced with transnasal insertion of an ultrathin endoscope. Preparation for EGD was performed in the same manner for all cases, as follows. Initially, the patient was provided a solution containing dimethicone and pronase as premedication to improve mucosal visibility, and naphazoline nitrate spray was applied to both the nostrils to prevent nasal edema and hemorrhage. Next, local anesthesia with $2 \%$ lidocaine gel was administered to the nostrils, and a pretreatment nasal catheter (14 or 16 Fr; Fujifilm, Tokyo, Japan) coated with lidocaine gel was gently inserted into either nostril so that the patient would not feel pain until the beginning of the exam- ination. All procedures were done without administration of scopolamine butylbromide or glucagon. None of the enrolled patients received any premedication or sedation before or during the procedure. Written informed consent was obtained from patients prior to the transnasal EGD procedure. The study protocol was approved by the ethics committee of Izumo City General Medical Center (registration number: 29-10).

\section{Statistical analysis}

The sample size for the clinical study was determined according to the findings of a pilot study. We adopted an objective indicator for the volume of washing solution used in a single endoscopic examination. In the pilot study, the mean amount of washing water used was $9.1 \mathrm{~mL}$. We predicted that use of the Cleash solution would result in a decrease in washing solution volume used during the examination of approximately $20 \%$ as compared to distilled water. A sample size of at least 485 patients per group was calculated to be necessary for a statistically significant difference between the groups in order to obtain a test power greater than $80 \%$ with a significance level of 0.05 in a two-tailed test.

Statistical analyses to compare the groups in each study were performed with a $\chi^{2}$ test, Mann-Whitney $U$ test, Friedman test, and Kruskal-Wallis rank test. Analysis with the Kruskal-Wallis rank test was done only when the Friedman test showed a significant difference. Categorical data were compared with either Student's $t$-test or Welch's test. $P$-values of less than 0.05 were considered significant.

\section{RESULTS}

\section{Study I}

The results of the present in vitro study of the cleaning effects after pork grease-soiling on the endoscope lens are shown in Fig. 2. The image quality of the photographs in the C-group was consistently better than that of the other groups. Furthermore, the C-group had visual quality maintained at a high level even when the soiling and cleaning procedures were repeated. In contrast, the visual quality of the images obtained in the $\mathrm{D}$ - and $\mathrm{W}$-group showed marked deterioration when the soiling and cleaning procedures were repeated.

\section{Study II}

The characteristics of the enrolled patients in the clinical study are shown in Table 1 . The C- and D-groups each contained 498 patients. No statistically significant differences were found between the groups for categorical data, including age, sex, duodenum observation rate, and numbers of biopsy and 


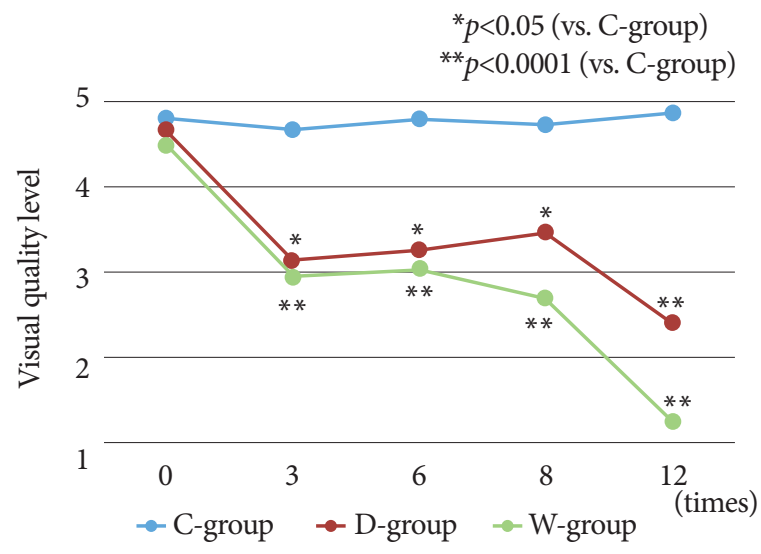

Fig. 2. The effect of various cleaning solutions on pork grease-soiled lens. The effect of the $5 \%$ Cleash solution (C) was consistently superior to that of the $5 \%$ dimethicone solution (D) and distilled water (W). Furthermore, visual quality remained high with $(C)$ even with repeated soiling and cleaning procedures.

Table 1. Study II Patient Characteristics

\begin{tabular}{lcc}
\hline & C-group & W-group \\
\hline Mean age, yr & $54.1 \pm 13.6$ & $53.5 \pm 12.0$ \\
Male : Female & $222: 276$ & $244: 254$ \\
Duodenum observation rate (\%) & 100 & 100 \\
$\begin{array}{l}\text { Endoscopic procedures } \\
\text { Biopsy, } n(\%)\end{array}$ & $19(3.8)$ & $18(3.6)$ \\
Chromoendoscopy, $n(\%)$ & $57(11.4)$ & $60(12.0)$ \\
\hline
\end{tabular}

chromoendoscopy cases. There were no critical complications or difficulties with the endoscopic systems experienced during any of the examinations. When (C) was used, no obstruction of the spray nozzle caused by the dimethicone solution occurred. Moreover, there was no adverse reaction between $(\mathrm{C})$ and any of the disposable materials, such as biopsy forceps or spraying catheter.

Volumes of washing solution used for lens cleaning in both groups are shown in Fig. 3. The mean volumes $( \pm \mathrm{SE})$ in the $\mathrm{C}$ - and $\mathrm{W}$-groups were $9.4 \pm 1.2$ and $11.6 \pm 1.5 \mathrm{~mL}$, respectively, and significantly lower in the C-group $(p<0.05)$. Stress levels reported by the endoscopists due to lens soiling are shown in Fig. 4 . The mean levels were $4.5 \pm 0.8$ and $3.9 \pm 1.1$, respectively, with the $\mathrm{C}$-group statistically superior. Cleanliness levels are shown in Fig. 5. Those mean values were $4.5 \pm 0.8$ and $4.0 \pm 1.0$, respectively, with the C-group again statistically superior to the $\mathrm{W}$-group.

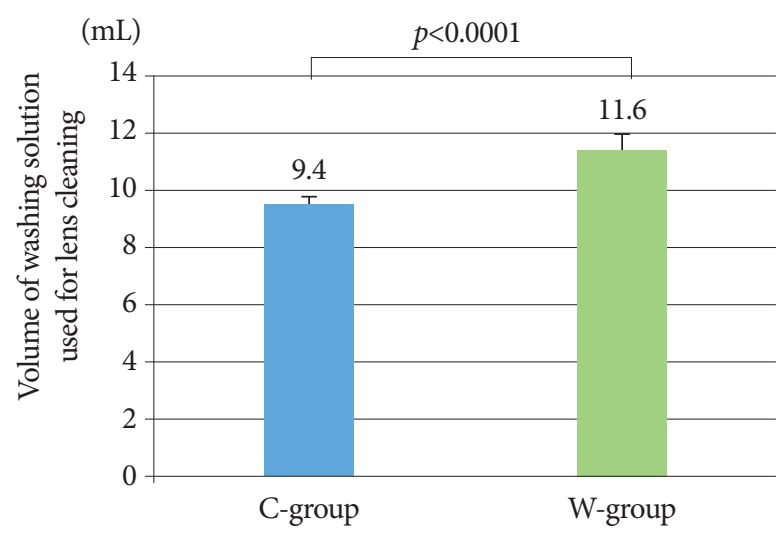

Fig. 3. Solution volume used for lens cleaning during esophagogastroduodenoscopy procedures. The one used in the C-group was significantly smaller as compared to the W-group $(p<0.05)$.

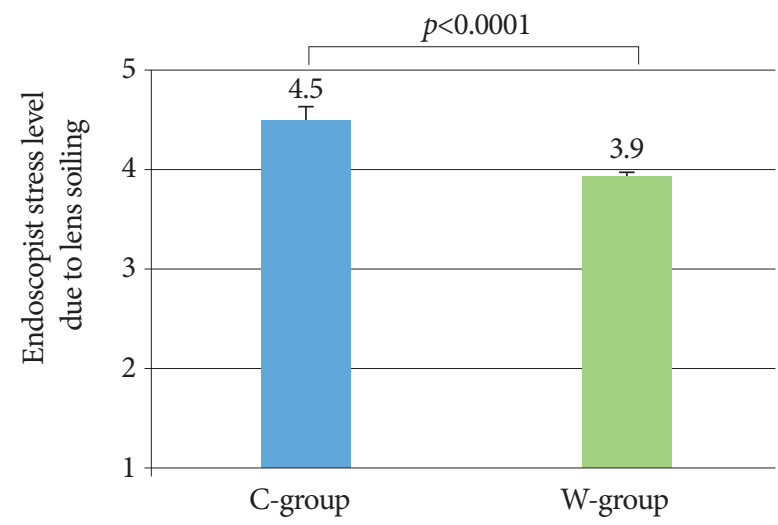

Fig. 4. Endoscopist stress level due to lens soiling during esophagogastroduodenoscopy procedures. The stress level from the C-group was lower compared to the W-group.

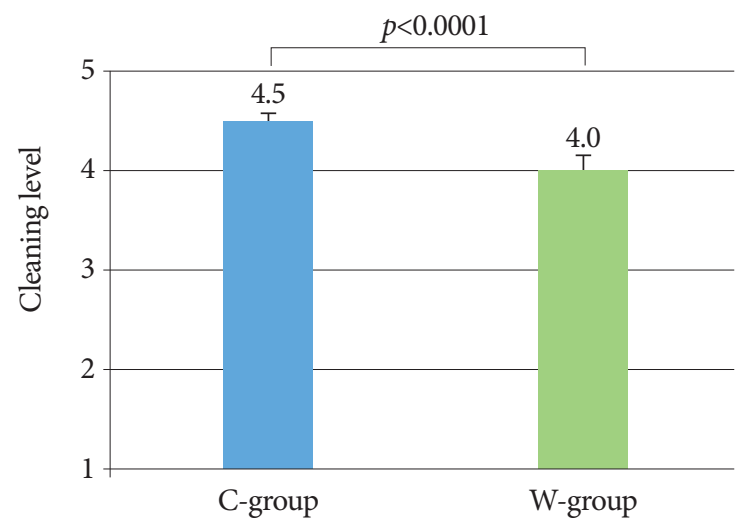

Fig. 5. The level of lens cleanliness during esophagogastroduodenoscopy. The level found in the C-group was significantly higher compared to the W-group. 


\section{DISCUSSION}

We previously reported the lens cleaning efficacy of oolong tea during transnasal EGD with a small-caliber endoscope. Oolong tea contains saponin, which has a surfactant effect advantageous for lens cleaning. ${ }^{13}$ However, the effect was limited, and we found lens soiling during small-caliber endoscopy to be a common concern in daily practice at our institution.

To obtain a higher level of lens cleaning, we used a washing liquid containing Cleash, a commercial lens cleaner, which involved consideration of 3 important issues. The first issue examined was the effect of Cleash on the endoscopic equipment and accessories. With the help of Fujifilm Co., we were able to confirm that there were no deleterious effects on the function of any of the endoscopic equipment or various accessories (snare, knife, injection needle, etc.). The second issue considered was the toxicity of this washing liquid. Yoshida et al. reported that no significant change in toxicity index was observed when model rats were administered Cleash at 2,000 $\mathrm{mg} / \mathrm{kg}$ of body weight and there was also no effect on gastric ulcers in those rats. ${ }^{11}$ In our previous investigation, we found that the amount of washing solution used during transnasal EGD ranged from 10 to $15 \mathrm{~mL}$, while in the present study, the amount of Cleash used ranged from 0.5 to $0.75 \mathrm{~mL}$ per examination. The amount of fatty acid polyglycerol esters in $0.75 \mathrm{~mL}$ of Cleash is $0.15 \mathrm{~g}$, while the limit for human toxicity has been estimated to be $20 \mathrm{~g}$ according to the Food Sanitation Department of Japan and basic research results. Thus, we considered that a $5 \%$ Cleash preparation may be safely used as a washing solution for patient examinations in the clinical study. The third issue was inconvenient bubbles created by the surfactant when spraying the washing solution containing Cleash onto an endoscope lens. In order to obtain a suitable cleaning effect, it was necessary to increase the concentration of Cleash. However, higher concentrations led to formation of more bubbles. Thus, we added dimethicone to suppress bubble formation, and carefully examined the best concentrations of Cleash and dimethicone prior to performing the present study. The results of an in vitro pilot study on the cleaning ability of $1 \%$, $2.5 \%$, and $5 \%$ Cleash solutions showed that lower concentrations were insufficient for cleaning. In addition, we examined the best concentration of dimethicone to suppress bubble formation when using a 5\% Cleash solution and concluded that the same amounts of each provided the best results. Thus, we produced a $5 \%$ Cleash solution by adding $10 \mathrm{~mL}$ of Cleash and $10 \mathrm{~mL}$ of dimethicone to $180 \mathrm{~mL}$ of distilled water in the tank.

Maintaining a clear endoscopic field of view is very important, not only for transnasal EGD with a small-caliber endo- scope, but also for transoral endoscopy with a conventional endoscope and therapeutic endoscopy procedures such as ESD. ${ }^{14,15}$ In Study I, a basic in vitro investigation, we used pork grease for soiling the endoscope lens. It is known that mucous soiling can be removed and cleansed by a water jet from the endoscopic nozzle because of its hydrophilic quality. On the other hand, lens soiling that is difficult to remove with a water jet alone is mainly caused by fats, proteins, and other components from residual food or body composition. Furthermore, since lens soiling encountered during ESD is caused by fats and proteins produced by the actions of the knife, we considered that pork grease would be suitable for our experiments in Study I. The 5\% Cleash solution significantly improved lens cleaning with the small-caliber endoscopes as compared with the $5 \%$ dimethicone solution and distilled water preparations when the soiling and cleaning procedures were repeated. Furthermore, the washing effect of the $5 \%$ Cleash solution was clearly superior as compared to oolong tea in our previous study. This effect is considered to be useful in clinical practice for transnasal small-caliber endoscopy examinations and may also be applied for therapeutic endoscopy procedures such as ESD. On the other hand, spray nozzle obstruction was often encountered with the 5\% dimethicone solution in contrast to the $5 \%$ Cleash solution, even though it contained the same amount of dimethicone, thus we speculated that Cleash provides a cleaning effect inside the nozzle. Indeed, no problems with obstruction occurred during the 3-month clinical research period or subsequent ongoing clinical use.

Based on our results, we consider that the present $5 \%$ Cleash solution provides a higher level of satisfaction in regard to lens cleaning and its superiority is the most important finding of this study. Although the volume of washing solution used for lens cleaning and endoscopist stress level may not be suitable for direct evaluation of lens cleaning effect, they are considered to be valid surrogate markers in association with the better endoscopic view provided by use of the $5 \%$ Cleash solution.

This study had some limitations. First, though the volume of washing solution used for lens cleaning may be affected by individual patient conditions (mucus in the nasal cavity, residue in the stomach, etc.), the methods for nasal anesthesia and gastric preparation were performed in the same manner for all cases, nevertheless, the condition of the present patients were relatively uniform. Second, endoscopist stress level and lens cleanliness level are indicators measured in a subjective manner. It is possible that accuracy may be improved by counting the number of lens cleanings and recording endoscopy time as objective indicators. However, as shown in Figs. 3, 4, and 5, the standard deviation was small and the present indicators were considered useful for the present evaluation. 
Recently, Yoshida et al. reported findings showing that a very low concentration $(0.5 \%)$ of Cleash solution improved water droplets adhering to an endoscope lens. ${ }^{12}$ However, in our experience, we think that it is difficult to obtain sufficient cleaning effect with such a low concentration. Furthermore, they reported a unique lens cleaning method using Cleash during colonic ESD in which the endoscopic hood is pressed against the mucosa to create an enclosed space, then, a stock solution of Cleash is injected via the endoscopic channel and maintained for 30 seconds. ${ }^{11}$ This method is not easy to apply and the amount of Cleash used is greater than the one used in the present study, in which a $5 \%$ solution was simply placed in the tank of the device. In our study, the amount of Cleash used in the gastrointestinal tract is small and the level of safety is high. As for the cost, the average amount of Cleash used for each examination was $0.58 \mathrm{ml}$, thus the cost was approximately 12.2 yen per endoscopic examination, which we concluded to be good cost performance. Furthermore, we consider that the present method may be effective for therapeutic endoscopy procedures, such as ESD, based on our ongoing clinical findings.

Maintaining a clear endoscopic view is very important not only for transnasal EGD, but also for therapeutic endoscopy procedures such as ESD, to provide accurate diagnosis as well as manipulation. The present $5 \%$ Cleash solution was found to be superior to distilled water for lens cleaning and good visibility was obtained during EGD with a transnasal small-caliber endoscope. Furthermore, the method is very convenient and highly safe, and may contribute to improving the accuracy of endoscopic diagnosis and therapy.

\section{Conflicts of Interest}

The authors have no potential conflicts of interest.

$$
\begin{gathered}
\text { Funding } \\
\text { None. }
\end{gathered}
$$

\section{Acknowledgments}

We thank all the staff of the Endoscopy Center of Izumo City General Medical Center for their help with this study.

\author{
Author Contributions \\ Conceptualization: Yoshinori Komazawa \\ Data curation: YKom, Mika Yuki, Nobuhiko Fukuba, Yoshiya Kobayashi, \\ Hitomi Ishitobi, Sayaka Nakashima, Makoto Nagaoka, Yoshiko Taka- \\ hashi, Toshihiro Shizuku \\ Formal analysis: MY \\ Methodology: YKom \\ Project administration: YKom \\ Writing-original draft: YKom
}

\section{ORCID}

Yoshinori Komazawa:

https://orcid.org/0000-0002-4002-3245
Mika Yuki:

Nobuhiko Fukuba:

Yoshiya Kobayashi:

Hitomi Ishitobi:

Sayaka Nakashima:

Makoto Nagaoka:

Yoshiko Takahashi:

Toshihiro Shizuku: https://orcid.org/0000-0002-0270-1447 https://orcid.org/0000-0003-3559-9490 https://orcid.org/0000-0003-4069-1478 https://orcid.org/0000-0002-0703-9405 https://orcid.org/0000-0003-1031-1585 https://orcid.org/0000-0001-9583-5761 https://orcid.org/0000-0003-1112-0617 https://orcid.org/0000-0003-3701-7458

\section{REFERENCES}

1. Yuki M, Amano Y, Komazawa Y, et al. Unsedated transnasal small-caliber esophagogastroduodenoscopy in elderly and bedridden patients. World J Gastroenterol 2009;15:5586-5591.

2. Yagi J, Adachi K, Arima N, et al. A prospective randomized comparative study on the safety and tolerability of transnasal esophagogastroduodenoscopy. Endoscopy 2005;37:1226-1231.

3. Kawai T, Miyazaki I, Yagi K, et al. Comparison of the effects on cardiopulmonary function of ultrathin transnasal versus normal diameter transoral esophagogastroduodenoscopy in Japan. Hepatogastroenterology 2007;54:770-774.

4. Kobayashi Y, Komazawa Y, Yuki M, et al. Use of anticoagulant or antiplatelet agents is not related to epistaxis in patients undergoing transnasal endoscopy. Endosc Int Open 2018;6:E104-E110.

5. Stroppa I, Grasso E, Paoluzi OA, et al. Unsedated transnasal versus transoral sedated upper gastrointestinal endoscopy: a one-series prospective study on safety and patient acceptability. Dig Liver Dis 2008;40:767-775.

6. Ai ZL, Lan CH, Fan LL, et al. Unsedated transnasal upper gastrointestinal endoscopy has favorable diagnostic effectiveness, cardiopulmonary safety, and patient satisfaction compared with conventional or sedated endoscopy. Surg Endosc 2012;26:3565-3572.

7. Komazawa Y, Amano Y, Yuki M, et al. Oolong tea is useful for lens cleansing in transnasal small-caliber esophagogastroduodenoscopy. Endoscopy 2010;42:104-108.

8. Tanuma T, Morita Y, Doyama H. Current status of transnasal endoscopy worldwide using ultrathin videoscope for upper gastrointestinal tract. Dig Endosc 2016;28(Suppl 1):25-31.

9. Hayashi Y, Yamamoto Y, Suganuma T, et al. Comparison of the diagnostic utility of the ultrathin endoscope and the conventional endoscope in early gastric cancer screening. Dig Endosc 2009;21:116-121.

10. Horiuchi A, Nakayama Y, Hidaka N, Ichise Y, Kajiyama M, Tanaka N. Prospective comparison between sedated high-definition oral and unsedated ultrathin transnasal esophagogastroduodenoscopy in the same subjects: pilot study. Dig Endosc 2009;21:24-28.

11. Yoshida N, Naito Y, Hirose R, et al. Risk of lens cloudiness during colorectal endoscopic submucosal dissection and ability of a novel lens cleaner to maintain and restore endoscopic view. Dig Endosc 2015;27:609-617.

12. Yoshida N, Naito Y, Yasuda R, et al. A novel lens cleaner to prevent water drop adhesions during colonoscopy and esophagogastroduodenoscopy. Endosc Int Open 2017;5:E1235-E1241.

13. Song S, Zhu L, Zhou W. Simultaneous removal of phenanthrene and cadmium from contaminated soils by saponin, a plant-derived biosurfactant. Environ Pollut 2008;156:1368-1370.

14. Tatsumi Y, Harada A, Matsumoto T, Tani T, Nishida H. Current status and evaluation of transnasal esophagogastroduodenoscopy. Dig Endosc 2009;21:141-146

15. Saito Y, Uraoka T, Yamaguchi Y, et al. A prospective, multicenter study of 1111 colorectal endoscopic submucosal dissections (with video). Gastrointest Endosc 2010;72:1217-1225. 